\title{
A Dual-Petrov-Galerkin Method for the Kawahara-Type Equations
}

\author{
Juan-Ming Yuan · Jie Shen · Jiahong Wu
}

Received: 19 February 2007 / Revised: 31 July 2007 / Accepted: 6 September 2007 /

Published online: 9 December 2007

(C) Springer Science+Business Media, LLC 2007

\begin{abstract}
An efficient and accurate numerical scheme is proposed, analyzed and implemented for the Kawahara and modified Kawahara equations which model many physical phenomena such as gravity-capillary waves and magneto-sound propagation in plasmas. The scheme consists of dual-Petrov-Galerkin method in space and Crank-Nicholson-leap-frog in time such that at each time step only a sparse banded linear system needs to be solved. Theoretical analysis and numerical results are presented to show that the proposed numerical is extremely accurate and efficient for Kawahara type equations and other fifth-order nonlinear equations.
\end{abstract}

Keywords Dual-Petrov-Galerkin · Fifth-order KDV equation · Kawahara equation · Legendre polynomials $\cdot$ Spectral approximation $\cdot$ Solitary waves ·

Oscillatory solitary waves

\section{Introduction}

Fifth-order Korteweg-de Vries type equations

$$
u_{t}-u_{x x x x x}=F\left(x, t, u, u_{x}, u_{x x}, u_{x x x}\right),
$$

This work is partially supported by the National Science Council of the Republic of China under the grant NSC 94-2115-M-126-004 and 95-2115-M-126-003.

This work is partially supported by NSF grant DMS-0610646.

J.-M. Yuan ( $\varangle)$

Department of Applied Mathematics, Providence University, Shalu, Taichung 433, Taiwan

e-mail: jmyuan@pu.edu.tw

J. Shen

Department of Mathematics, Purdue University, West Lafayette, IN 47907, USA

e-mail: shen@math.purdue.edu

J. Wu

Department of Mathematics, Oklahoma State University, Stillwater, OK 74078, USA

e-mail: jiahong@math.okstate.edu 
arise naturally in modeling many wave phenomena. Although most of the study for this type of equations are focused on the Cauchy problem or initial and periodic boundary-value problem, there are at least two compelling reasons to study the initial and boundary-value problems: (i) some applications, such as a wave maker, are naturally set on a semi-infinite interval; (ii) for computational purpose, one often has to reduce the problem in an infinite domain to a finite domain. Hence, we shall focus our attention on the following initial and boundary-value problem:

$$
\begin{aligned}
& \alpha v_{t}+\mu v_{x}+\gamma v^{p} v_{x}+\beta v_{x x x}-v_{x x x x x}=0, \quad x \in(-1,1), t \in(0, T], \\
& v(-1, t)=g(t), \quad v_{x}(-1, t)=h(t), \quad v(1, t)=v_{x}(1, t)=v_{x x}(1, t)=0, \quad t \in[0, T], \\
& v(x, 0)=v_{0}(x), \quad x \in(-1,1),
\end{aligned}
$$

where $\alpha \geq 0, \mu, \beta$ and $\gamma$ rescaled parameters depending on the physical parameters and scaling. In particular, $\beta$ is related the Bond number for water waves in the presence of surface tension and $\beta=0$ corresponds to the critical Bond number $\frac{1}{3}$ (cf. for instance, [21]). When $p=1$, this equation is called the Kawahara equation and when $p=2$, the modified Kawahara equation [11]. The Kawahara type equations have been derived to model magneto-acoustic waves in plasmas [11] and shallow water waves with surface tension [9]. With $g(t)=h(t)=0$, one can regard (1.2) as an approximation to the initial-value problem before the wave reaches the boundaries; while with $g(t), h(t) \neq 0$ one can view (1.2) as an approximate model for the initial and boundary-value problem on a quarter-plane before the wave reaches the right boundary. Although we will only deal with (1.2) for the sake of simplicity, it will become clear that our approaches, in particular the numerical algorithms, can also be applied to other fifth-order KDV equations.

Due to the fifth-order terms in (1.2), it is very difficult to compute the solutions of these equations accurately and efficiently. Recently, a new dual-Petrov-Galerkin method for third and higher odd-order equations is proposed and has proven to be very effective for the KdV equation in a bounded domain [18] and in semi-infinite intervals [19]. Hence, we shall adopt the dual-Petrov-Galerkin method for the fifth-order equation (1.2). However, besides the difficulty associated with the fifth-order term, (1.2) is more difficult to handle analytically and numerically than the $\mathrm{KdV}$ equation due to the facts that (i) for $\beta<0$, solutions of (1.2) exhibit highly oscillatory behaviors, inducing considerable analytical and numerical difficulties; (ii) the nonlinearity in (1.2) with $p>1$ is much stronger than that in the KdV equation.

The main purposes of this paper are to apply the dual-Petrov-Galerkin method proposed in [18] to (1.2), to provide a rigorous error analysis for it and to demonstrate its effectiveness by computing some computationally challenging solitary and oscillatory solitary waves. To fix the idea, we have chosen a set of commonly used boundary conditions in (1.2). Other set of boundary conditions can be handled in a similar fashion as elucidated in Remark 3.2.

The rest of the paper is organized as follows. In Sect. 2, we describe the dual-PetrovGalerkin method for a linear, time-independent fifth-order equation. In Sect. 3, we study a fully discretization of the Kawahara-type equation. In Sect. 4, we present some numerical results for the Kawahara-type equations to illustrate the accuracy and efficiency of our algorithm.

We now introduce some notations. We shall use the weighted Sobolev spaces $H_{\omega}^{m}(\Omega)$ $(m=0, \pm 1, \ldots)$ whose norms are denoted by $\|\cdot\|_{m, \omega}$. In particular, the norm and inner product of $L_{\omega}^{2}(\Omega)=H_{\omega}^{0}(\Omega)$ are denoted by $\|\cdot\|_{\omega}$ and $(\cdot, \cdot)_{\omega}$ respectively. Let $\omega(x)$ be a 
positive function (not necessarily in $L^{1}(\Omega)$ ), we define

$$
L_{\omega}^{2}(\Omega)=\left\{u:(u, u)_{\omega}:=\int_{\Omega} u^{2}(x) \omega(x) d x<+\infty\right\}
$$

with the norm $\|\cdot\|_{\omega}=(u, u)_{\omega}^{\frac{1}{2}}$. We denote by $c$ a generic constant that is independent of any parameters and functions. In most cases, we shall simply use the expression $A \lesssim B$ to mean that there exists a generic constant $c$ such that $A \leq c B$.

\section{Dual-Petrov-Galerkin Method for a Fifth-Order Equation}

In this section we recall the dual-Petrov-Galerkin method [18] for a linear fifth-order equation and summarize some of the estimates that we will need in the next section.

Consider the following boundary-value problem (BVP)

$$
\begin{aligned}
& \alpha u+\beta u_{x x x}-u_{x x x x x}=f, \quad x \in I=(-1,1), \\
& u( \pm 1)=u_{x}( \pm 1)=u_{x x}(1)=0
\end{aligned}
$$

where $\alpha$ and $\beta$ are given constants. Without loss of generality, we only consider homogenous boundary conditions, for non-homogenous boundary conditions can be easily handled by considering $v=u-\hat{u}$, where $\hat{u}$ is the unique quartic polynomial satisfying the nonhomogenous boundary conditions.

We start with a few notations. For any constants $\alpha$ and $\beta$, let $\omega^{\alpha, \beta}(x)=(1-x)^{\alpha}(1+x)^{\beta}$ be the Jacobi weight function with index $(\alpha, \beta)$. We define a set of non-uniformly weighted Sobolev spaces as follows:

$$
H_{\omega^{\alpha, \beta}}^{m}(I)=\left\{u \in L_{\omega^{\alpha, \beta}}^{2}(I): \partial_{x}^{l} u \in L_{\omega^{l-\alpha, l-\beta}}^{2}(I), 1 \leq l \leq m\right\} .
$$

Let $P_{N}$ denote the space of polynomials of degree $\leq N$ and set

$$
\begin{aligned}
& W_{N}=\left\{u \in P_{N}: u( \pm 1)=u_{x}( \pm 1)=u_{x x}(1)=0\right\}, \\
& W_{N}^{*}=\left\{u \in P_{N}: u( \pm 1)=u_{x}( \pm 1)=u_{x x}(-1)=0\right\} .
\end{aligned}
$$

We consider the following dual-Petrov-Galerkin approximation for (2.1): Find $u_{N} \in W_{N}$ such that

$$
\alpha\left(u_{N}, \eta_{N}\right)-\beta\left(\partial_{x}^{2} u_{N}, \partial_{x} \eta_{N}\right)+\left(\partial_{x}^{2} u_{N}, \partial_{x}^{3} \eta_{N}\right)=\left(f, \eta_{N}\right), \quad \forall \eta_{N} \in W_{N}^{*} .
$$

Notice that for any $v_{N} \in W_{N}$ we have $\omega^{-1,1} v_{N} \in W_{N}^{*}$. Thus, the above dual-PetrovGalerkin formulation is equivalent to the following weighted spectral-Galerkin approximation: Find $u_{N} \in W_{N}$ such that

$$
\begin{aligned}
& \alpha\left(u_{N}, v_{N}\right)_{\omega^{-1,1}}-\beta\left(\partial_{x}^{2} u_{N}, \omega^{1,-1} \partial_{x}\left(v_{N} \omega^{-1,1}\right)\right)_{\omega^{-1,1}}+\left(\partial_{x}^{2} u_{N}, \omega^{1,-1} \partial_{x}^{3}\left(v_{N} \omega^{-1,1}\right)\right)_{\omega^{-1,1}} \\
& \quad=\left(f, v_{N}\right)_{\omega^{-1,1}}, \quad \forall v_{N} \in W_{N},
\end{aligned}
$$

where $(u, v)_{\omega^{-1,1}}=\int_{I} u v \omega^{-1,1} d x$. As it will become clear below (see also [18]), the dualPetrov-Galerkin formulation (2.4) is most suitable for implementation while the weighted Galerkin formulation (2.5) is more convenient for error analysis. 
As suggested in $[16,17]$, one should choose compact combinations of orthogonal polynomials as basis functions to minimize the bandwidth and the condition number of the coefficient matrix corresponding to (2.5). Let $\left\{p_{k}\right\}$ be a sequence of orthogonal polynomials. As a general rule, for one-dimensional differential equations with $m$ boundary conditions, one should look for basis functions in the form

$$
\Phi_{k}(x)=p_{k}(x)+\sum_{j=1}^{m} a_{j}^{(k)} p_{k+j}(x),
$$

where $a_{j}^{(k)}(j=1, \ldots, m)$ are chosen so that $\phi_{k}(x)$ satisfy the $m$ homogeneous boundary conditions.

Let $L_{k}$ be the $k$ th degree Legendre polynomial (cf. [23]) which is mutually orthogonal in $L^{2}(-1,1)$, i.e.

$$
\int_{-1}^{1} L_{k}(x) L_{j}(x) d x=\frac{2}{2 k+1} \delta_{k j} .
$$

By setting $p_{k}=L_{k}$ in (2.6), it is easy to verify that, for $N \geq 5$, we have

$$
\begin{aligned}
& W_{N}=\operatorname{span}\left\{\Phi_{0}, \Phi_{1}, \ldots, \Phi_{N-5}\right\}, \\
& W_{N}^{*}=\operatorname{span}\left\{\Psi_{0}, \Psi_{1}, \ldots, \Psi_{N-5}\right\}
\end{aligned}
$$

with

$$
\begin{aligned}
\Phi_{k}= & L_{k}-\frac{2 k+3}{2 k+7} L_{k+1}-\frac{4 k+10}{2 k+7} L_{k+2}+\frac{4 k+6}{2 k+9} L_{k+3}+\frac{2 k+3}{2 k+7} L_{k+4} \\
& -\frac{4 k^{2}+16 k+15}{(2 k+7)(2 k+9)} L_{k+5}, \\
\Psi_{k}= & L_{k}+\frac{2 k+3}{2 k+7} L_{k+1}-\frac{4 k+10}{2 k+7} L_{k+2}-\frac{4 k+6}{2 k+9} L_{k+3}+\frac{2 k+3}{2 k+7} L_{k+4} \\
& +\frac{4 k^{2}+16 k+15}{(2 k+7)(2 k+9)} L_{k+5} .
\end{aligned}
$$

It can be checked that $\left\{\Phi_{k}\right\}_{k=0}^{\infty}$ and $\left\{\Psi_{k}\right\}_{k=0}^{\infty}$ form a complete orthogonal basis in $L_{\omega^{-3,-2}}^{2}$ and $L_{\omega^{-2,-3}}^{2}$, respectively [7].

Now, let $\Pi_{N}=\Pi_{N}^{(-3,-2)}$ be the $L_{\omega^{-3,-2}}^{2}$-orthogonal projector: $L_{\omega^{-3,-2}}^{2} \rightarrow W_{N}$ defined by

$$
\left(u-\Pi_{N} u, v_{N}\right)_{\omega^{-3,-2}}=0, \quad \forall v_{N} \in W_{N} .
$$

It is important to note that $\Pi_{N}$ also satisfies the following property:

$$
\left(\partial_{x}^{2}\left(u-\Pi_{N} u\right), \partial_{x}^{3} v_{N}\right)=\left(u-\Pi_{N} u, \omega^{3,2} \partial_{x}^{5} v_{N}\right)_{\omega^{-3,-2}}=0, \quad \forall u \in H_{\omega^{-3,-2}}^{2}, \quad v_{N} \in W_{N}^{*}
$$

We now recall some results established in [18].

First of all, we have the following estimates on the projection error.

\section{Theorem 2.1}

$$
\left\|\partial_{x}^{l}\left(u-\Pi_{N} u\right)\right\|_{\omega^{l-3, l-2}} \lesssim N^{l-m}\left\|\partial_{x}^{m} u\right\|_{\omega^{m-3, m-2},}, \quad \forall u \in H_{\omega^{-3,-2}}^{m}, \quad 0 \leq l \leq m .
$$


Next, we recall two Hardy-type inequalities:

Lemma 2.1 Let $V_{N}=\left\{u \in P_{N}: u( \pm 1)=u_{x}(1)=0\right\}$, we have

$$
\begin{aligned}
& \int_{I} \frac{u^{2}}{(1-x)^{4}} d x \leq \frac{4}{9} \int_{I} \frac{u_{x}^{2}}{(1-x)^{2}} d x, \quad \forall u \in V_{N}, \\
& \int_{I} \frac{u^{2}}{(1-x)^{3}} d x \leq \int_{I} \frac{u_{x}^{2}}{1-x} d x, \quad \forall u \in V_{N} .
\end{aligned}
$$

The following result is essential for the well-posedness of our dual-Petrov-Galerkin method.

\section{Lemma 2.2}

$$
\begin{aligned}
& \frac{1}{3}\left\|u_{x}\right\|_{\omega^{-2,0}}^{2} \leq\left(u_{x},\left(u \omega^{-1,1}\right)_{x x}\right) \leq 3\left\|u_{x}\right\|_{\omega^{-2,0}}^{2}, \quad \forall u \in V_{N} . \\
& \frac{5}{7} \int_{I} \frac{u_{x x}^{2}}{(1-x)^{2}} d x \leq\left(\partial_{x}^{2} u, \partial_{x}^{3}\left(u \omega^{-1,1}\right)\right) \leq \frac{203}{15} \int_{I} \frac{u_{x x}^{2}}{(1-x)^{2}} d x, \quad \forall u \in W_{N} .
\end{aligned}
$$

Finally, the following results can be proven by a usual argument using the above estimates [18]:

Theorem 2.2 Let $u$ be the solution of (2.1) and assume $u \in H_{\omega^{-3,-2}}^{m}$. Then, for any $\alpha, \beta>0$, the problem (2.4) admits a unique solution $u_{N}$ which satisfies the following error estimate:

$$
\begin{aligned}
& \sqrt{\alpha}\left\|e_{N}\right\|_{\omega^{-1,1}}+\sqrt{\beta} N^{-1}\left\|\left(e_{N}\right)_{x}\right\|_{\omega^{-2,0}}+N^{-2}\left\|\left(e_{N}\right)_{x x}\right\|_{\omega^{-1,0}} \\
& \quad \lesssim(1+\sqrt{\beta} N) N^{-m}\left\|\partial_{x}^{m} u\right\|_{\omega^{m-3, m-2}}, \quad m \geq 2 .
\end{aligned}
$$

Let us denote

$$
\begin{array}{lc}
u_{N}=\sum_{k=0}^{N-5} \tilde{u}_{k} \Phi_{k}, & \bar{u}=\left(\tilde{u}_{0}, \tilde{u}_{1}, \ldots, \tilde{u}_{N-5}\right)^{t}, \\
\tilde{f}_{k}=\left(f, \Psi_{k}\right), & \bar{u}=\left(\tilde{f}_{0}, \tilde{f}_{1}, \ldots, \tilde{f}_{N-5}\right)^{t}, \\
m_{i j}=\left(\Phi_{j}, \Psi_{i}\right), & M=\left(m_{i j}\right)_{i, j=0,1, \ldots, N-5}, \\
p_{i j}=-\left(\Phi_{j}^{\prime \prime}, \Psi_{i}^{\prime}\right), & P=\left(p_{i j}\right)_{i, j=0,1, \ldots, N-5}, \\
s_{i j}=\left(\Phi_{j}^{\prime \prime}, \Psi_{i}^{\prime \prime \prime}\right), & S=\left(s_{i j}\right)_{i, j=0,1, \ldots, N-5 .}
\end{array}
$$

Then, the variational formulation (2.4) leads to the following linear system

$$
(\alpha M+\beta P+S) \bar{u}=\bar{f} .
$$

By using integration by parts and orthogonal properties of Legendre polynomials, one easily determines that

$$
m_{i j}=0 \quad \text { for }|i-j|>5, \quad p_{i j}=0 \quad \text { for }|i-j|>2, \quad s_{i j}=0 \quad \text { for } i \neq j .
$$

Hence, the linear system (2.16) can be easily and efficiently inverted. 


\section{Applications to the Kawahara-Type Equation}

We now apply the dual-Petrov-Galerkin method to the fifth-order equation (1.2). To this end, we first reformulate (1.2) to an equivalent problem with homogeneous boundary conditions. Let $\hat{v}(x, t)=\frac{(1-x)^{3}}{8}\left[\left(h(t)+\frac{3}{2} g(t)\right)(x+1)+g(t)\right]$ and write $v(x, t)=u(x, t)+\hat{v}(x, t)$. Then, for $p=1, u$ satisfies the following equation with homogeneous boundary conditions:

$$
\begin{aligned}
& \alpha u_{t}+a(x, t) u+b(x, t) u_{x}+\gamma u^{p} u_{x}+\beta u_{x x x}-u_{x x x x x}=f, \quad x \in(-1,1), t \in(0, T], \\
& u( \pm 1, t)=u_{x}( \pm 1, t)=u_{x x}(1, t)=0, \quad t \in[0, T], \\
& u(x, 0)=u_{0}(x)=v_{0}(x)-\hat{v}(x, 0), \quad x \in(-1,1),
\end{aligned}
$$

where $a(x, t)=\gamma \hat{v}_{x}, b(x, t)=\mu+\gamma \hat{v}(x, t)$ and $f(x, t)=-\alpha \hat{v}_{t}(x, t)-\mu \hat{v}_{x}-\beta \hat{v}_{x x x}-$ $\gamma \hat{v}^{p} \hat{v}_{x}$.

Although (3.1) is equivalent to (1.2) only when $p=1$, we will still study (3.1) for all $p \geq 1$, since for $p>1$, (3.1) retains the term $u^{p} u_{x}$ which has the highest nonlinearity in the reformulated equation. Therefore, even some lower order linear and nonlinear terms in the reformulated equation are dropped out in (3.1), it is clear that our analysis would carry over even if those terms are included in (3.1).

For a given $\Delta t$, we set $t_{k}=k \Delta t$ and let $u_{N}^{0}=\Pi_{N} u_{0}$ and $u_{N}^{1} \in V_{N}$ be an appropriate approximation of $u\left(\cdot, t_{1}\right)$, for instance, we can compute $u_{N}^{1}$ using one step of a semi-implicit first-order scheme so that for $u \in C^{3}\left(0, T ; L_{\omega^{2,2}}^{2}(I)\right) \cap C^{1}\left(0, T ; H_{\omega^{-3,-2}}^{m}(I)\right)$, we have

$$
\left\|u_{N}^{1}-\Pi_{N} u\left(\cdot, t_{1}\right)\right\|_{\omega^{-1,1}} \lesssim \Delta t^{2}+(1+|\beta| N) N^{-m}
$$

Then, the second-order Crank-Nicolson leap-frog scheme in time with a weighted Galerkin approximation in space reads: For $k=1,2, \ldots,[T / \Delta t]-1$, find $u_{N}^{k+1} \in W_{N}$ such that

$$
\begin{aligned}
& \frac{\alpha}{2 \Delta t}\left(u_{N}^{k+1}-u_{N}^{k-1}, v_{N}\right)_{\omega^{-1,1}}+\frac{\beta}{2}\left(\partial_{x}\left(u_{N}^{k+1}+u_{N}^{k-1}\right), \partial_{x}^{2}\left(v_{N} \omega^{-1,1}\right)\right) \\
& \quad+\frac{1}{2}\left(\partial_{x}^{2}\left(u_{N}^{k+1}+u_{N}^{k-1}\right), \partial_{x}^{3}\left(v_{N} \omega^{-1,1}\right)\right) \\
& =\left(f\left(\cdot, t_{k}\right), v_{N}\right)_{\omega^{-1,1}}-\left(a u_{N}^{k}, v_{N}\right)_{\omega^{-1,1}}+\left(u_{N}^{k}, \partial_{x}\left(b v_{N} \omega^{-1,1}\right)\right) \\
& \quad+\frac{\gamma}{p+1}\left(\left(u_{N}^{k}\right)^{p+1}, \partial_{x}\left(v_{N} \omega^{-1,1}\right)\right), \quad \forall v_{N} \in W_{N} .
\end{aligned}
$$

It is clear that at each time step, (3.3) reduces to (2.5) which can be efficiently solved.

In order to prove the stability and convergence of (3.3), we shall first consider a modified scheme. To this end, let $M$ be such that $|u(x, t)| \leq M$ for $x \in[-1,1]$ and $t \in[0, T]$. We define a cut-off function

$$
H(x)= \begin{cases}x, & |x| \leq 2 M, \\ 2 M, & x>2 M, \\ -2 M, & x<-2 M .\end{cases}
$$

It is easy to verify that

$$
|H(x)-H(y)| \leq|x-y|, \quad \forall x, y .
$$


Then, the modified Crank-Nicolson leap-frog dual-Petrov-Galerkin approximation is: For $k=1,2, \ldots,[T / \Delta t]-1$, find $u_{N}^{k+1} \in W_{N}$ such that

$$
\begin{aligned}
& \frac{\alpha}{2 \Delta t}\left(u_{N}^{k+1}-u_{N}^{k-1}, v_{N}\right)_{\omega^{-1,1}}+\frac{\beta}{2}\left(\partial_{x}\left(u_{N}^{k+1}+u_{N}^{k-1}\right), \partial_{x}^{2}\left(v_{N} \omega^{-1,1}\right)\right) \\
& \quad+\frac{1}{2}\left(\partial_{x}^{2}\left(u_{N}^{k+1}+u_{N}^{k-1}\right), \partial_{x}^{3}\left(v_{N} \omega^{-1,1}\right)\right) \\
& =\left(f\left(\cdot, t_{k}\right), v_{N}\right)_{\omega^{-1,1}}+\frac{\gamma}{p+1}\left(H\left(u_{N}^{k}\right)^{p} u_{N}^{k}, \partial_{x}\left(v_{N} \omega^{-1,1}\right)\right) \\
& \quad-\left(a u_{N}^{k}, v_{N}\right)_{\omega^{-1,1}}+\left(u_{N}^{k}, \partial_{x}\left(b v_{N} \omega^{-1,1}\right)\right), \quad \forall v_{N} \in W_{N} .
\end{aligned}
$$

We denote $\hat{e}_{N}^{k}=\Pi_{N} u\left(\cdot, t_{k}\right)-u_{N}^{k}, \tilde{e}_{N}^{k}=u\left(\cdot, t_{k}\right)-\Pi_{N} u\left(\cdot, t_{k}\right)$ and $e_{N}^{k}=u\left(\cdot, t_{k}\right)-u_{N}^{k}$.

Theorem 3.1 We assume that (3.1) admits a unique solution $u \in C^{3}\left(0, T ; L_{\omega^{2,2}}^{2}(I)\right) \cap$ $C^{1}\left(0, T ; H_{\omega^{-3,-2}}^{m}(I)\right)$ with $m \geq 3$. Then, for $\alpha>0$ and $\beta>-\frac{15}{112}$, the scheme (3.6) is unconditionally stable and the following error estimates hold for $1 \leq n \leq[T / \Delta t]-1$ :

$$
\begin{aligned}
\left\|e_{N}^{n+1}\right\|_{\omega^{-1,1}} & \lesssim \Delta t^{2}+(1+|\beta| N) N^{-m} \\
\left(\Delta t \sum_{k=1}^{n}\left\|\partial_{x}^{2}\left(e_{N}^{k+1}+e_{N}^{k-1}\right)\right\|_{\omega^{-1,0}}^{2}\right)^{\frac{1}{2}} & \lesssim \Delta t^{2}+(1+|\beta| N) N^{1-m} .
\end{aligned}
$$

Remark 3.1 The condition on $\beta$ appears to be a natural one, since the global existence of (1.2) can only be established under some similar limitations on $\beta$ (cf., for instance, [5]).

Proof Let $E^{k}(k=1,2, \ldots)$ be the truncation error defined by

$$
\begin{gathered}
\frac{\alpha}{2 \Delta t}\left(u\left(\cdot, t_{k+1}\right)-u\left(\cdot, t_{k-1}\right)\right)+a\left(\cdot, t_{k}\right) u\left(\cdot, t_{k}\right)+b\left(\cdot, t_{k}\right) u_{x}\left(\cdot, t_{k}\right)+\gamma u^{p}\left(\cdot, t_{k}\right) \partial_{x} u\left(\cdot, t_{k}\right) \\
+\frac{\beta}{2} \partial_{x}^{3}\left(u\left(\cdot, t_{k+1}\right)+u\left(\cdot, t_{k-1}\right)\right)-\frac{1}{2} \partial_{x}^{5}\left(u\left(\cdot, t_{k+1}\right)+u\left(\cdot, t_{k-1}\right)\right)-f\left(\cdot, t_{k}\right)=E^{k}(\cdot) .
\end{gathered}
$$

Comparing (3.6) with (3.7) and using (2.11), we have

$$
\begin{aligned}
& \frac{\alpha}{2 \Delta t}\left(\hat{e}_{N}^{k+1}-\hat{e}_{N}^{k-1}, v_{N}\right)_{\omega^{-1,1}}+\frac{1}{2}\left(\partial_{x}^{2}\left(\hat{e}_{N}^{k+1}+\hat{e}_{N}^{k-1}\right), \partial_{x}^{3}\left(v_{N} \omega^{-1,1}\right)\right) \\
& =\frac{\gamma}{p+1}\left(u^{p+1}\left(\cdot, t_{k}\right)-H\left(u_{N}^{k}\right)^{p} u_{N}^{k}, \partial_{x}\left(v_{N} \omega^{-1,1}\right)\right)-\left(a\left(\hat{e}_{N}^{k}+\tilde{e}_{N}^{k}\right), v_{N}\right)_{\omega^{-1,1}} \\
& \quad+\left(\left(\hat{e}_{N}^{k}+\tilde{e}_{N}^{k}\right), \partial_{x}\left(b v_{N} \omega^{-1,1}\right)\right)+\left(E^{k}, v_{N}\right)_{\omega^{-1,1}}-\frac{\alpha}{2 \Delta t}\left(\tilde{e}_{N}^{k+1}-\tilde{e}_{N}^{k-1}, v_{N}\right)_{\omega^{-1,1}} \\
& \quad-\frac{\beta}{2}\left(\partial_{x}\left(\hat{e}_{N}^{k+1}+\hat{e}_{N}^{k-1}+\tilde{e}_{N}^{k+1}+\tilde{e}_{N}^{k-1}\right), \partial_{x}^{2}\left(v_{N} \omega^{-1,1}\right)\right):=R H S\left(v_{N}\right), \quad \forall v_{N} \in V_{N} .
\end{aligned}
$$

Let $A=\max _{x \in[-1,1], t \in[0, T]}|a(x, t)|$ and $B=\max _{x \in[-1,1], t \in[0, T]}\left(|b(x, t)|+\left|\partial_{x} b(x, t)\right|\right)$. We now take $v_{N}=2 \Delta t\left(\hat{e}_{N}^{k+1}+\hat{e}_{N}^{k-1}\right)$ in (3.8), thanks to Lemma 2.2, we have

$$
\alpha\left(\left\|\hat{e}_{N}^{k+1}\right\|_{\omega^{-1,1}}^{2}-\left\|\hat{e}_{N}^{k-1}\right\|_{\omega^{-1,1}}^{2}\right)+\frac{5 \Delta t}{7}\left\|\partial_{x}^{2}\left(\hat{e}_{N}^{k+1}+\hat{e}_{N}^{k-1}\right)\right\|_{\omega^{-2,0}}^{2} \leq R H S\left(2 \Delta t\left(\hat{e}_{N}^{k+1}+\hat{e}_{N}^{k-1}\right)\right) .
$$


Below, we shall bound the right-hand side terms using repeatedly the Cauchy-Schwarz inequality, Lemma 2.1 and the crude estimate

$$
\begin{gathered}
\left\|\partial_{x} v_{N}\right\|_{\omega^{-2,0}}^{2} \leq 4\left\|\partial_{x} v_{N}\right\|_{\omega^{-4,0}}^{2} \leq \frac{16}{9}\left\|\partial_{x}^{2} v_{N}\right\|_{\omega^{-2,0}}^{2}, \\
-2 \Delta t\left(a\left(\hat{e}_{N}^{k}+\tilde{e}_{N}^{k}\right), \hat{e}_{N}^{k+1}+\hat{e}_{N}^{k-1}\right)_{\omega^{-1,1}} \leq A \Delta t\left(\left\|\tilde{e}_{N}^{k}+\hat{e}_{N}^{k}\right\|_{\omega^{-1,1}}^{2}+\left\|\hat{e}_{N}^{k+1}+\hat{e}_{N}^{k-1}\right\|_{\omega^{-1,1}}^{2}\right) .
\end{gathered}
$$

Given $\delta>0$ which can be arbitrarily small,

$$
\begin{aligned}
2 \Delta t\left(E^{k}, \hat{e}_{N}^{k+1}+\hat{e}_{N}^{k-1}\right)_{\omega^{-1,1}} & \leq 2 \Delta t\left\|E^{k}\right\|_{\omega^{2,2}}\left\|\hat{e}_{N}^{k+1}+\hat{e}_{N}^{k-1}\right\|_{\omega^{-4,0}} \\
& \leq c \Delta t\left\|E^{k}\right\|_{\omega^{2,2}}^{2}+4 \delta \Delta t\left\|\partial_{x}\left(\hat{e}_{N}^{k+1}+\hat{e}_{N}^{k-1}\right)\right\|_{\omega^{-2,0}}^{2} \\
& \leq c \Delta t\left\|E^{k}\right\|_{\omega^{2,2}}^{2}+\delta \Delta t\left\|\partial_{x}^{2}\left(\hat{e}_{N}^{k+1}+\hat{e}_{N}^{k-1}\right)\right\|_{\omega^{-2,0}}^{2}
\end{aligned}
$$

Let us denote $\tilde{E}_{N}^{k}=\frac{1}{2 \Delta t}\left(\tilde{e}_{N}^{k+1}-\tilde{e}_{N}^{k-1}\right)$. Similarly as above, we have

$$
-\alpha\left(\tilde{e}_{N}^{k+1}-\tilde{e}_{N}^{k-1}, \hat{e}_{N}^{k+1}+\hat{e}_{N}^{k-1}\right)_{\omega^{-1,1}} \leq c \Delta t\left\|\tilde{E}_{N}^{k}\right\|_{\omega^{2,2}}^{2}+\delta \Delta t\left\|\partial_{x}^{2}\left(\hat{e}_{N}^{k+1}+\hat{e}_{N}^{k-1}\right)\right\|_{\omega^{-2,0}}^{2} .
$$

By using (3.5), the identity $a^{p}-b^{p}=(a-b)\left(\sum_{i=0}^{p-1} a^{i} b^{p-1-i}\right)$ and the assumption $|u(x, t)| \leq M$ for all $x$ and $t$, we have

$$
\begin{aligned}
\left|u^{p+1}\left(\cdot, t_{k}\right)-H\left(u_{N}^{k}\right)^{p} u_{N}^{k}\right| & =\left|u\left(\cdot, t_{k}\right)\left(H\left(u\left(\cdot, t_{k}\right)\right)^{p}-H\left(u_{N}^{k}\right)^{p}\right)+H\left(u_{N}^{k}\right)^{p}\left(u\left(\cdot, t_{k}\right)-u_{N}^{k}\right)\right| \\
& \leq(p+1) M\left|u\left(\cdot, t_{k}\right)-u_{N}^{k}\right| \leq(p+1) M\left(\left|\tilde{e}_{N}^{k}\right|+\left|\hat{e}_{N}^{k}\right|\right) .
\end{aligned}
$$

We now use the identity

$$
\partial_{x}\left(v_{N} \omega^{-1,1}\right)=\partial_{x} v_{N} \omega^{-1,1}+2 v_{N} \omega^{-2,0}
$$

to split the first term in $R H S\left(v_{N}\right)$ into two terms and estimate them separately as follows:

$$
\begin{aligned}
\gamma \Delta & t\left(u^{p+1}\left(\cdot, t_{k}\right)-H\left(u_{N}^{k}\right)^{p} u_{N}^{k}, \partial_{x}\left(\hat{e}_{N}^{k+1}+\hat{e}_{N}^{k-1}\right) \omega^{-1,1}\right) \\
& \leq|\gamma| \Delta t\left\|u^{p+1}\left(\cdot, t_{k}\right)-H\left(u_{N}^{k}\right)^{p} u_{N}^{k}\right\|_{\omega^{0,2}}\left\|\partial_{x}\left(\hat{e}_{N}^{k+1}+\hat{e}_{N}^{k-1}\right)\right\|_{\omega^{-2,0}} \\
& \leq c \Delta t\left(\left\|\tilde{e}_{N}^{k}\right\|_{\omega^{-2,-1}}^{2}+\left\|\hat{e}_{N}^{k}\right\|_{\omega^{-1,1}}^{2}\right)+\delta \Delta t\left\|\partial_{x}^{2}\left(\hat{e}_{N}^{k+1}+\hat{e}_{N}^{k-1}\right)\right\|_{\omega^{-2,0}}^{2} .
\end{aligned}
$$

We recall the following inequality [18],

$$
\int_{I} \phi^{2} \omega^{-3,-1} d x \leq 4 \int_{I}\left(\phi_{x}\right)^{2} \omega^{-1,0} d x
$$

which holds for all $\phi$ such that $\phi( \pm 1)=0$ and $\int_{I}\left(\phi_{x}\right)^{2} \omega^{-1,0} d x<\infty$. Thanks to Lemma 2.1 and (3.10),

$$
\begin{aligned}
\gamma & \Delta t \\
& \left(u^{p+1}\left(\cdot, t_{k}\right)-H\left(u_{N}^{k}\right)^{p} u_{N}^{k},\left(\hat{e}_{N}^{k+1}+\hat{e}_{N}^{k-1}\right) \partial_{x} \omega^{-1,1}\right) \\
& \leq 2|\gamma| \Delta t\left\|u^{p+1}\left(\cdot, t_{k}\right)-H\left(u_{N}^{k}\right)^{p} u_{N}^{k}\right\|_{\omega^{-1,1}}\left\|\left(\hat{e}_{N}^{k+1}+\hat{e}_{N}^{k-1}\right)\right\|_{\omega^{-3,-1}} \\
& \leq c \Delta t\left(\left\|\tilde{e}_{N}^{k}\right\|_{\omega^{-2,-1}}^{2}+\left\|\hat{e}_{N}^{k}\right\|_{\omega^{-1,1}}^{2}\right)+\delta \Delta t\left\|\partial_{x}^{2}\left(\hat{e}_{N}^{k+1}+\hat{e}_{N}^{k-1}\right)\right\|_{\omega^{-2,0}}^{2} .
\end{aligned}
$$

Similarly, we have

$$
\begin{aligned}
& \Delta t\left(\left(\hat{e}_{N}^{k}+\tilde{e}_{N}^{k}\right), \partial_{x}\left(b\left(\hat{e}_{N}^{k+1}+\hat{e}_{N}^{k-1}\right) \omega^{-1,1}\right)\right)=\Delta t\left(\left(\hat{e}_{N}^{k}+\tilde{e}_{N}^{k}\right), b \partial_{x}\left(\hat{e}_{N}^{k+1}+\hat{e}_{N}^{k-1}\right) \omega^{-1,1}\right) \\
& \quad+\Delta t\left(\left(\hat{e}_{N}^{k}+\tilde{e}_{N}^{k}\right), b\left(\hat{e}_{N}^{k+1}+\hat{e}_{N}^{k-1}\right) \partial_{x} \omega^{-1,1}\right)+\Delta t\left(\left(\hat{e}_{N}^{k}+\tilde{e}_{N}^{k}\right), \partial_{x} b\left(\hat{e}_{N}^{k+1}+\hat{e}_{N}^{k-1}\right) \omega^{-1,1}\right) \\
& \leq c B \Delta t\left(\left\|\tilde{e}_{N}^{k}\right\|_{\omega^{-2,-1}}^{2}+\left\|\hat{e}_{N}^{k}\right\|_{\omega^{-1,1}}^{2}\right)+\delta \Delta t\left\|\partial_{x}^{2}\left(\hat{e}_{N}^{k+1}+\hat{e}_{N}^{k-1}\right)\right\|_{\omega^{-2,0}}^{2} .
\end{aligned}
$$


It remains to estimate the last term in $R H S\left(v_{N}\right)$. By the first inequality in Lemma 2.2, we have that for $\beta \geq 0$,

$$
\Delta t \beta\left(\partial_{x}\left(\hat{e}_{N}^{k+1}+\hat{e}_{N}^{k-1}\right), \partial_{x}^{2}\left(\left(\hat{e}_{N}^{k+1}+\hat{e}_{N}^{k-1}\right) \omega^{-1,1}\right)\right) \geq \frac{1}{3} \Delta t \beta\left\|\partial_{x}\left(\hat{e}_{N}^{k+1}+\hat{e}_{N}^{k-1}\right)\right\|_{\omega^{-2,0}}^{2},
$$

and for $\beta<0$,

$$
\begin{aligned}
\Delta t\left|\beta\left(\partial_{x}\left(\hat{e}_{N}^{k+1}+\hat{e}_{N}^{k-1}\right), \partial_{x}^{2}\left(\left(\hat{e}_{N}^{k+1}+\hat{e}_{N}^{k-1}\right) \omega^{-1,1}\right)\right)\right| & \leq 3 \Delta t|\beta|\left\|\partial_{x}\left(\hat{e}_{N}^{k+1}+\hat{e}_{N}^{k-1}\right)\right\|_{\omega^{-2,0}}^{2} \\
& \leq \frac{16}{3} \Delta t|\beta|\left\|\partial_{x}^{2}\left(\hat{e}_{N}^{k+1}+\hat{e}_{N}^{k-1}\right)\right\|_{\omega^{-2,0}}^{2} .
\end{aligned}
$$

Noticing the identity

$$
\partial_{x}^{2}\left(v_{N} \omega^{-1,1}\right)=\partial_{x}^{2} v_{N} \omega^{-1,1}+4 \partial_{x} v_{N} \omega^{-2,0}+4 v_{N} \omega^{-3,0}
$$

and by Hölder's inequality, we have

$$
\begin{aligned}
& \left|\left(\partial_{x}\left(\tilde{e}_{N}^{k+1}+\tilde{e}_{N}^{k-1}\right), \partial_{x}^{2}\left(v_{N} \omega^{-1,1}\right)\right)\right| \leq\left\|\partial_{x}\left(\tilde{e}_{N}^{k+1}+\tilde{e}_{N}^{k-1}\right)\right\|_{\omega^{-2,-1}}\left\|\partial_{x}^{2} v_{N}\right\|_{\omega^{0,3}} \\
& \quad+4\left\|\partial_{x}\left(\tilde{e}_{N}^{k+1}+\tilde{e}_{N}^{k-1}\right)\right\|_{\omega^{-2,-1}}\left\|\partial_{x} v_{N}\right\|_{\omega^{-2,1}}+4\left\|\partial_{x}\left(\tilde{e}_{N}^{k+1}+\tilde{e}_{N}^{k-1}\right)\right\|_{\omega^{-2,-1}}\left\|v_{N}\right\|_{\omega^{-4,1}}
\end{aligned}
$$

By Lemma 2.1, $\left\|v_{N}\right\|_{\omega^{-4,1}} \leq 2\left\|v_{N}\right\|_{\omega^{-4,0}} \leq \frac{4}{3}\left\|\partial_{x} v_{N}\right\|_{\omega^{-2,0}}$. Therefore,

$$
\begin{aligned}
& \Delta t\left|\beta\left(\partial_{x}\left(\tilde{e}_{N}^{k+1}+\tilde{e}_{N}^{k-1}\right), \partial_{x}^{2}\left(\left(\hat{e}_{N}^{k+1}+\hat{e}_{N}^{k-1}\right) \omega^{-1,1}\right)\right)\right| \\
& \quad \leq C|\beta| \Delta t\left\|\partial_{x}\left(\tilde{e}_{N}^{k+1}+\tilde{e}_{N}^{k-1}\right)\right\|_{\omega^{-2,-1}}\left(\left\|\partial_{x}\left(\hat{e}_{N}^{k+1}+\hat{e}_{N}^{k-1}\right)\right\|_{\omega^{-2,0}}+\left\|\partial_{x}^{2}\left(\hat{e}_{N}^{k+1}+\hat{e}_{N}^{k-1}\right)\right\|_{\omega^{-2,0}}\right) \\
& \quad \leq C \Delta t\left\|\partial_{x}\left(\tilde{e}_{N}^{k+1}+\tilde{e}_{N}^{k-1}\right)\right\|_{\omega^{-2,-1}}^{2}+\delta \Delta t\left\|\partial_{x}^{2}\left(\hat{e}_{N}^{k+1}+\hat{e}_{N}^{k-1}\right)\right\|_{\omega^{-2,0}}^{2} .
\end{aligned}
$$

Combining the above inequalities into (3.9), we obtain

$$
\begin{aligned}
\alpha\left(\left\|\hat{e}_{N}^{k+1}\right\|_{\omega^{-1,1}}^{2}-\left\|\hat{e}_{N}^{k-1}\right\|_{\omega^{-1,1}}^{2}\right)+\left(\frac{5}{7}-4 \delta+\frac{16 \min (\beta, 0)}{3}\right) \Delta t\left\|\partial_{x}^{2}\left(\hat{e}_{N}^{k+1}+\hat{e}_{N}^{k-1}\right)\right\|_{\omega^{-2,0}}^{2} \\
\leq c \Delta t\left(\left\|E^{k}\right\|_{\omega^{2,2}}^{2}+\left\|\tilde{E}_{N}^{k}\right\|_{\omega^{2,2}}^{2}+\left\|\hat{e}_{N}^{k+1}\right\|_{\omega^{-1,1}}^{2}+\left\|\hat{e}_{N}^{k}\right\|_{\omega^{-1,1}}^{2}\right. \\
\left.\quad+\left\|\hat{e}_{N}^{k-1}\right\|_{\omega^{-1,1}}^{2}+\left\|\tilde{e}_{N}^{k}\right\|_{\omega^{-2,-1}}^{2}+|\beta|\left\|\partial_{x}\left(\tilde{e}_{N}^{k+1}+\tilde{e}_{N}^{k-1}\right)\right\|_{\omega^{-2,-1}}^{2}\right) .
\end{aligned}
$$

For $\beta>-\frac{15}{112}$, we can choose $\delta$ sufficiently small such that $\frac{5}{7}-4 \delta+\frac{16 \min (\beta, 0)}{3}>0$. We can then apply the standard discrete Gronwall lemma to the above inequality to get, for any $1 \leq n \leq[T / \Delta t]-1$,

$$
\begin{aligned}
& \alpha\left\|\hat{e}_{N}^{n+1}\right\|_{\omega^{-1,1}}^{2}+\left\|\partial_{x}^{2}\left(\hat{e}_{N}^{k+1}+\hat{e}_{N}^{k-1}\right)\right\|_{\omega^{-2,0}}^{2} \lesssim\left\|\hat{e}_{N}^{0}\right\|_{\omega^{-1,1}}^{2}+\left\|\hat{e}_{N}^{1}\right\|_{\omega^{-1,1}}^{2} \\
& \quad+\Delta t \sum_{k=1}^{n}\left(\left\|\tilde{e}_{N}^{k}\right\|_{\omega^{-2,-1}}^{2}+\left\|E^{k}\right\|_{\omega^{2,2}}^{2}+\left\|\tilde{E}_{N}^{k}\right\|_{\omega^{2,2}}^{2}+|\beta|\left(\left\|\partial_{x} \tilde{e}_{N}^{k+1}\right\|_{\omega^{-2,-1}}^{2}+\left\|\partial_{x} \tilde{e}_{N}^{k-1}\right\|_{\omega^{-2,-1}}^{2}\right)\right) .
\end{aligned}
$$

We can finally conclude by using the triangular inequality, (3.2), the regularity assumptions and Theorem 2.1. 
Now, using the same argument in [18], we can show that the following statement holds.

Corollary 3.1 Under the conditions of Theorem 3.1, there exists $c_{0}$ such that for $\Delta t N \leq c_{0}$, the two schemes (3.6) and (3.3) are equivalent.

Remark 3.2 It is clear from the above discussion that other sets of boundary conditions can be handled similarly. For example, if we replace the boundary condition in (3.1) by

$$
u(-1, t)=0, \quad u(1, t)=u_{x}(1, t)=u_{x x}(1, t)=u_{x x x}(1, t)=0,
$$

then, we should replace $W_{N}$ and $W_{N}^{*}$ in (2.3) by

$$
\begin{aligned}
& W_{N}=\left\{u \in P_{N}: u(-1)=0, u(1)=u_{x}(1)=u_{x x}(1)=u_{x x x}(1)=0\right\}, \\
& W_{N}^{*}=\left\{u \in P_{N}: u(-1)=u_{x}(-1)=u_{x x}(-1)=u_{x x x}(-1)=0, u(1)=0\right\},
\end{aligned}
$$

and we have $W_{N} \in L_{\omega^{-4,-1}}^{2}(I)$ and $W_{N}^{*} \in L_{\omega^{-1,-4}}^{2}(I)$. Therefore, the results in Theorems 2.2 and 3.1 can be carried over to this case with essentially the same procedure.

\section{Numerical Results}

In this section, we present some numerical results for the Kawahara and modified Kawahara equations.

\subsection{Solitary Waves}

We consider first numerical approximations of solitary wave solutions for the Kawahara equation and modified Kawahara equation [20]. More precisely, we consider the Kawahara equation

$$
u_{t}+u u_{x}+u_{x x x}-u_{x x x x x}=0, \quad u(x, 0)=u_{e x}(x, 0),
$$

where

$$
u_{e x}(x, t)=\frac{105}{169} \operatorname{sech}^{4}\left(\frac{1}{2 \sqrt{13}}\left(x-\frac{36 t}{169}-x_{0}\right)\right)
$$

is an exact soliton solution of (4.1); and the modified Kawahara equation

$$
u_{t}+u_{x}+u^{2} u_{x}+p u_{x x x}+q u_{x x x x x}=0, \quad u(x, 0)=u_{e x}(x, 0),
$$

where

$$
u_{e x}(x, t)= \pm \frac{3 p}{\sqrt{-10 q}} \operatorname{sech}^{2}\left(\frac{1}{2} \sqrt{\frac{-p}{5 q}}\left(x-\frac{25 q-4 p^{2}}{25 q} t-x_{0}\right)\right)
$$

is an exact soliton solution of (4.3) and $p, q$ are two parameters.

In order to apply the dual-Petrov-Galerkin method, we fix $x_{0}=0$ and restrict the problem to the finite interval $[-L, L]$ with $L$ sufficiently large such that the solution $u_{e x}( \pm L, t)$, $\partial_{x} u_{e x}( \pm L, t), \partial_{x}^{2} u_{e x}(L, t)$ are essentially zero for $t \in[0, T]$ (where $T$ is given). We apply 
Table $1 L^{2}$-errors for solitary wave solutions in the Kawahara equation

\begin{tabular}{lllr}
\hline Time & $L^{2}$-error with $\Delta t=1.0 \mathrm{E}-4$ & $L^{2}$-error with $\Delta t=2.0 \mathrm{E}-4$ & Rate \\
\hline 0.5 & $3.44 \mathrm{E}-7$ & $1.374 \mathrm{E}-6$ & 3.99 \\
1.0 & $5.926 \mathrm{E}-7$ & $2.358 \mathrm{E}-6$ & 3.98 \\
2.0 & $1.104 \mathrm{E}-6$ & $4.389 \mathrm{E}-6$ & 3.98 \\
4.0 & $2.147 \mathrm{E}-6$ & $8.494 \mathrm{E}-6$ & 3.96 \\
\hline
\end{tabular}

Table $2 L^{2}$-errors for solitary wave solutions in the modified Kawahara equation

\begin{tabular}{llll}
\hline Time & $L^{2}$-error with $\Delta t=1.0 \mathrm{E}-4$ & $L^{2}$-error with $\Delta t=2.0 \mathrm{E}-4$ & Rate \\
\hline 0.1 & $1.77 \mathrm{E}-5$ & $7.071 \mathrm{E}-5$ & 4 \\
0.2 & $2.93 \mathrm{E}-5$ & $1.173 \mathrm{E}-4$ & 4 \\
0.4 & $5.19 \mathrm{E}-5$ & $2.076 \mathrm{E}-4$ & 4 \\
0.5 & $6.33 \mathrm{E}-5$ & $2.531 \mathrm{E}-4$ & 4 \\
\hline
\end{tabular}

the scaling $\tilde{x}=L^{-1} x, \tilde{t}=L^{-1} t$, and for the sake of simplicity, still use $(x, t)$ to denote $(\tilde{x}, \tilde{t})$. Then, we are led to consider the following scaled Kawahara equation

$$
\begin{aligned}
& u_{t}+u u_{x}+\frac{1}{L^{2}} u_{x x x}-\frac{1}{L^{4}} u_{x x x x x}=0, \quad x \in(-1,1), \\
& u( \pm 1)=u_{x}( \pm 1)=u_{x x}(1)=0, \\
& u(x, 0)=\frac{105}{169} \operatorname{sech}^{4}\left(\frac{L}{2 \sqrt{13}} x\right),
\end{aligned}
$$

and the modified Kawahara equation

$$
\begin{aligned}
& u_{t}+u_{x}+u^{2} u_{x}+\frac{1}{L^{2}} u_{x x x}-\frac{1}{L^{4}} u_{x x x x x}=0, \quad x \in(-1,1), \\
& u( \pm 1)=u_{x}( \pm 1)=u_{x x}(1)=0 \\
& u(x, 0)=\frac{3}{\sqrt{10}} \operatorname{sech}^{2}\left(\frac{L}{2} \sqrt{\frac{1}{5}} x\right) .
\end{aligned}
$$

Below, we present some numerical results with $L=200$ using $N=1000$ in the dualPetrov-Galerkin scheme. In Tables 1 and 2, we list the $L^{2}$-errors at different times with two different time steps. Note that in these numerical tests, the spatial error (with $N=1000$ ) is negligible and the error is dominated by the time discretization error. Tables 1 and 2 clearly indicates that the Crank-Nicholson-leap-frog scheme is of second-order in time. In Fig. 1, we plot the computed and exact solutions for the Kawahara and modified Kawahara equations. The computed and exact solutions are virtually indistinguishable.

\subsection{Oscillatory Solitary Waves}

We now consider the so called "oscillatory solitary waves" which consist of a packet of solitary waves with arbitrary small perturbations [9]. Oscillatory solitary waves can be found 

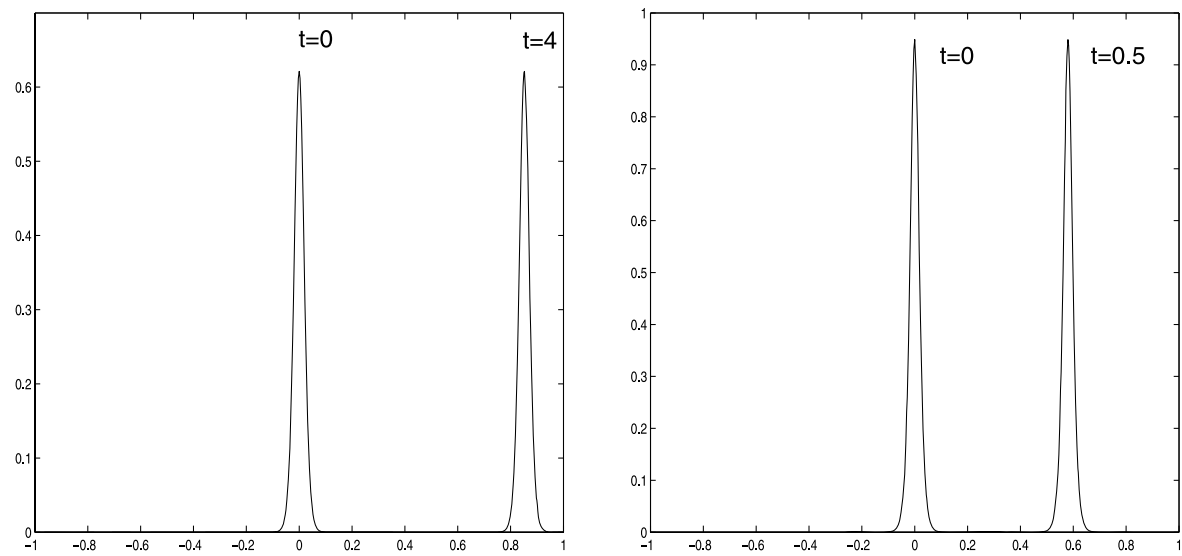

Fig. 1 Solitary wave solutions in the Kawahara (left) and modified Kawahara (right) equation

in the presence of surface tension with the Bond number lies between 0 and $\frac{1}{3}$ (cf., e.g. $[10,24]$ for numerical study and $[1,21,22]$ for analytical work). We note that in the fifthorder KDV equation which models the water wave in the presence of surface tension, the critical Bond number $\frac{1}{3}$ corresponds to $\beta=0$ in (3.1); the case with Bond number greater than (resp. smaller than) $\frac{1}{3}$ corresponds to $\beta>0$ (resp. $\beta<0$ ). Oscillatory solitary waves occur in (3.1) with $\beta<0$.

Following Kawahara [11], we consider the following Kawahara equation

$$
u_{t}+6 u u_{x}+u_{x x x}+\epsilon^{2} u_{x x x x x}=0,
$$

which, after a change of variable $\tilde{x}=-x$, corresponds to (3.1) with $\beta<0$. Hunter and Schedule [9] proposed (4.7) as a model equation for capillary-gravity waves when the bond number us just less than the critical value of $\frac{1}{3}$. Pomeau et al. [14] used techniques of asymptotics pioneered by Segur and Kruskal [15] to show that the amplitude of the tail oscillations is exponentially small with respect to the small parameter $\epsilon$. Kichenassamy and Olver [12] showed the nonexistence of solitary wave solutions of (4.7). The relationship between the tail amplitudes and the phase shifts can be found in [2,6].

By assuming that the solution of (4.7) takes the form of a small-amplitude modulated wave packet, we can find the following asymptotic solution for (4.7) by using two-scale expansion listed in [8] correct to $\mathrm{O}\left(\epsilon^{2}\right)$,

$$
\begin{aligned}
u_{e x}(x, t)= & \sqrt{\frac{2}{19}} \epsilon \cos \left(k_{m} \xi+\phi_{0}\right) \operatorname{sech} X+\epsilon^{2}\left\{\frac{187}{57 \sqrt{19}} \sin \left(k_{m} \xi+\phi_{0}\right) \operatorname{sech} X \tanh X\right. \\
& \left.-\frac{4}{19}\left(3+\frac{1}{3} \cos \left(2 k_{m} \xi+2 \phi_{0}\right)\right) \operatorname{sech}^{2} X\right\}+\mathrm{O}\left(\epsilon^{3}\right):=\bar{u}(x, t)+\mathrm{O}\left(\epsilon^{3}\right),
\end{aligned}
$$

where $\xi=x-c t$, and $X=\epsilon \xi$ represents the wave envelope. The constant $\phi_{0}$ controls the phase of the carrier oscillations relative to the peak of the envelop at $X=0$ [3, 4]. In [3], a split-step Fourier method [13] was used to solve the above equation.

In order to apply the dual-Petrov-Galerkin scheme, we rescale $(4.7)$ with $(\tilde{x}, \tilde{t})=$ $\left(-L^{-1} x, L^{-1} t\right)$, still use $(x, t)$ to denote $(\tilde{x}, \tilde{t})$, we are led to consider the following initial- 
Table 3 Differences between the computed solutions of (4.9) and the asymptotic solution with $\epsilon=0.01$ and $\Delta t=1.0 \mathrm{E}-5$

\begin{tabular}{lll}
\hline$t$ & $L_{2}$ & $L_{\infty}$ \\
\hline 0.05 & $1.57 \mathrm{E}-5$ & $6.9 \mathrm{E}-5$ \\
0.1 & $1.635 \mathrm{E}-5$ & $9.5 \mathrm{E}-5$ \\
0.2 & $1.776 \mathrm{E}-5$ & $8.4 \mathrm{E}-5$ \\
\hline
\end{tabular}
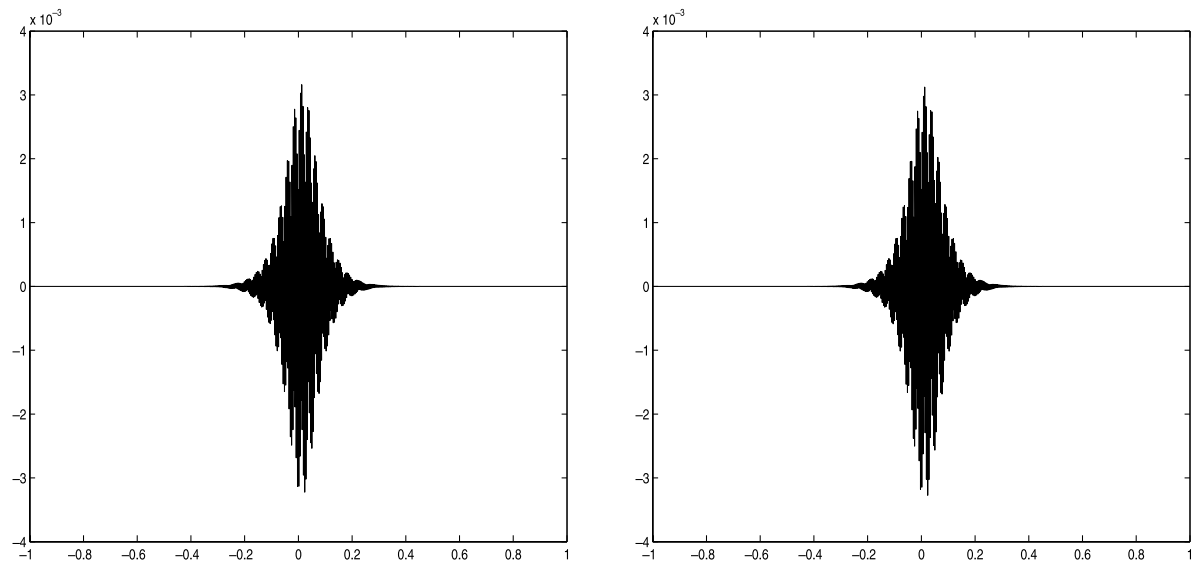

Fig. $2 \epsilon=0.01$ and $t=0.05$, asymptotic solution (left graph) and numerical solution (right graph)
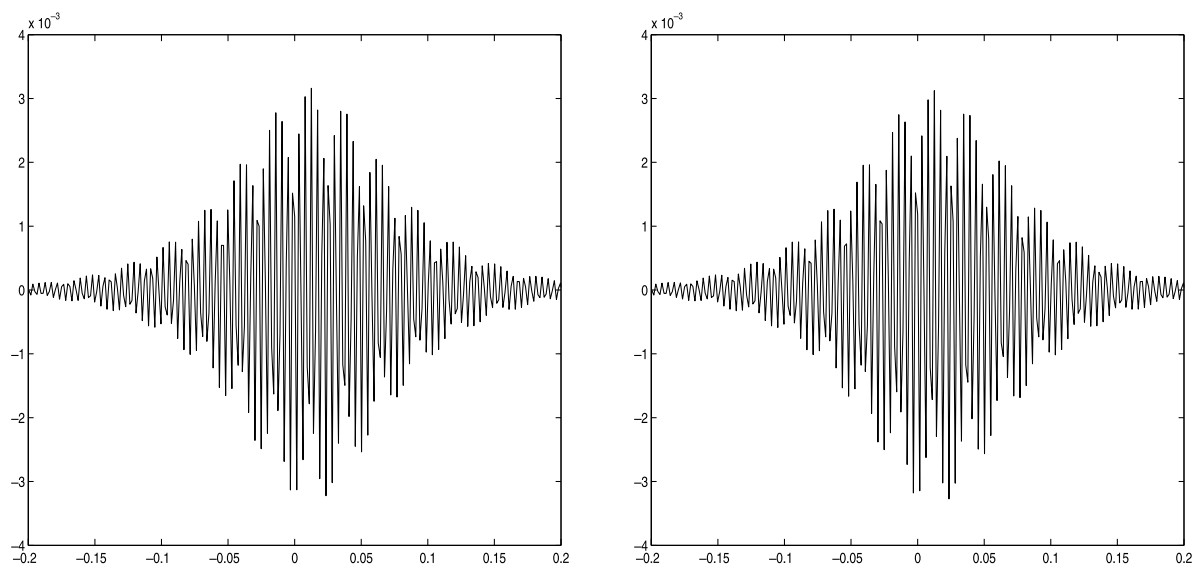

Fig. 3 Continued. $\epsilon=0.01$ and $t=0.05$, asymptotic solution (left graph) and numerical solution (right graph)

and boundary-value problem:

$$
\begin{aligned}
& u_{t}-6 u u_{x}-\frac{1}{L^{2}} u_{x x x}-\frac{1}{L^{4}} u_{x x x x x}=0, \quad x \in(-1,1) \\
& u( \pm 1, t)=u_{x}( \pm 1, t)=u_{x x}(1, t)=0, \quad u(x, 0)=\bar{u}\left(-L^{-1} x, 0\right) .
\end{aligned}
$$



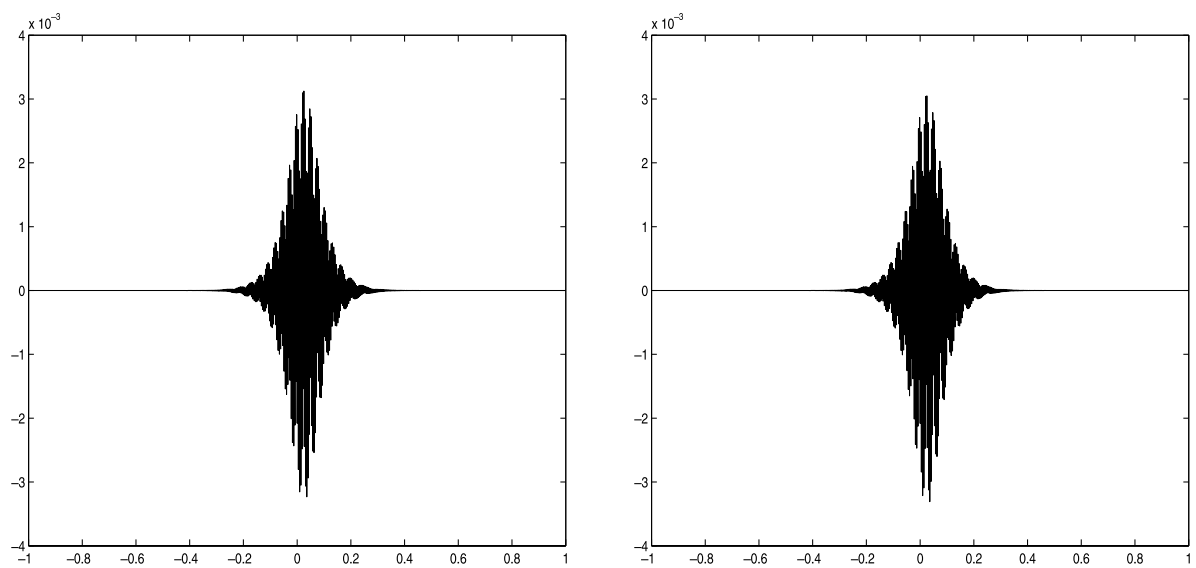

Fig. $4 \epsilon=0.01$ and $t=0.1$, asymptotic solution (left graph) and numerical solution (right graph)
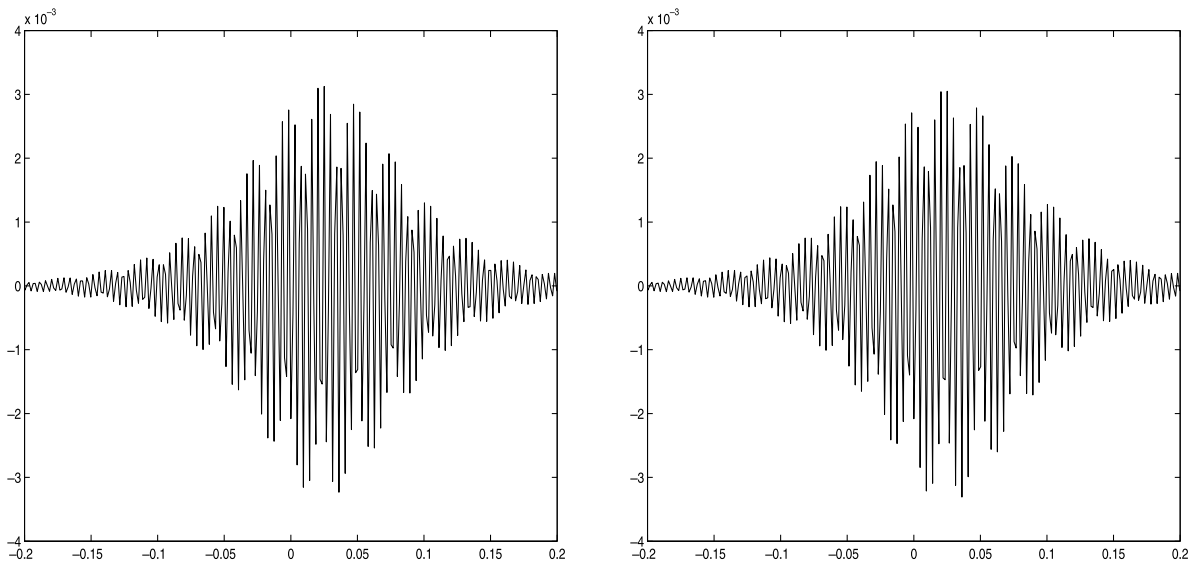

Fig. 5 Continued. $\epsilon=0.01$ and $t=0.1$, asymptotic solution (left graph) and numerical solution (right graph)

In our numerical experiments, we take $k_{m}=\sqrt{0.5}, \phi_{0}=0, \epsilon=0.01, \xi=x-0.25 t$ and $L=2000$. It can be checked from $(4.8,4.9)$ that with $\epsilon=0.01$ and $L=2000$, the boundary conditions in (4.9) are accurate at least to the order $\mathrm{O}\left(\epsilon^{3}\right)$ for $t \in(0,0.2)$ (which corresponds to the real time $t \in(0,400))$. Note that for smaller $\epsilon$, larger $L$ is needed to ensure that the boundary conditions in (4.9) are sufficiently accurate.

In all the computations presented below, we use $\Delta t=1.0 \mathrm{E}-5$ and $N=2000$. In Table 3 , we list the $L^{2}$ and $L^{\infty}$ errors between the computed solutions of (4.9) and the asymptotic solution at three different (scaled) times $t=0.05,0.1,0.2$ which correspond to original times $t=100,200,400$. Note that the accuracy is limited by the accuracy of the asymptotic solution which is accurate to the order of $\epsilon^{3}$.

In Figs. 2, 3, 4, 5, 6 and 7, we plot the computed solutions and the asymptotic solutions at three different times on the whole interval (Figs. 2, 4 and 6) and on a shorter interval (Figs. 3, 5 and 7). We notice that the solutions to (4.9) exhibit highly oscillatory behaviors which are 

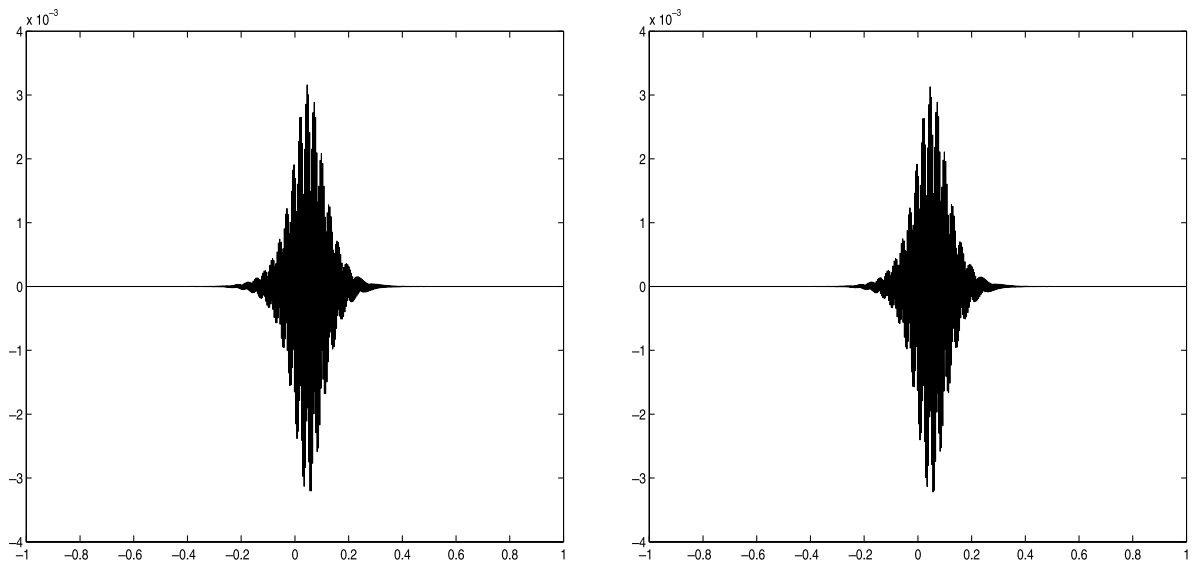

Fig. $6 \epsilon=0.01$ and $t=0.2$, asymptotic solution (left graph) and numerical solution (right graph)
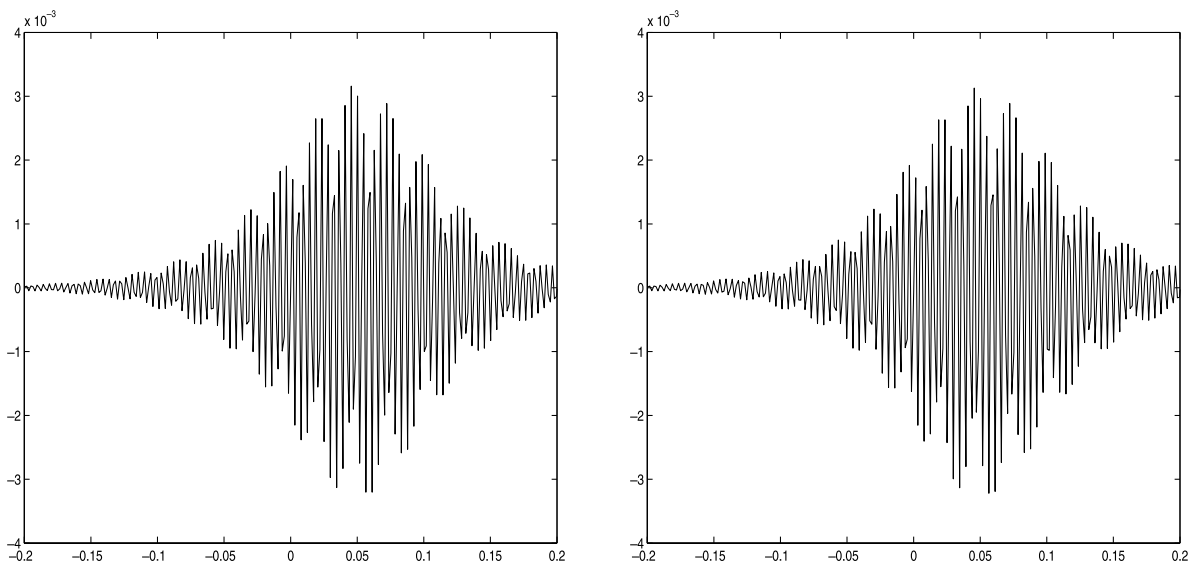

Fig. 7 Continued. $\epsilon=0.01$ and $t=0.2$, asymptotic solution (left graph) and numerical solution (right graph)

extremely difficult to compute $[4,11]$ but are well captured by our dual-Petrov-Galerkin method.

\section{Concluding Remarks}

We presented a numerical scheme consists of dual-Petrov-Galerkin method in space and Crank-Nicholson-leap-frog in time for the Kawahara and modified Kawahara equations which has been proposed to model many physical phenomena such as gravity-capillary waves and magneto-sound propagation in plasmas. At each time step, the scheme is reduced to a linear fifth-order equation with constant coefficients that can be very efficiently solved by the dual-Petrov-Galerkin method. It is shown that the scheme is stable under a very mild stability constraint, and is second-order accurate in time and spectrally accurate in space. We used this scheme to compute solitary wave solutions and oscillatory solitary 
wave solutions of the Kawahara and modified Kawahara equations, and our numerical results indicate that the scheme is capable of capturing, with very high accuracy, solitary wave solutions and highly oscillatory solutions with modest computational costs.

Acknowledgement The authors would like to thank Professor S.-M. Sun for fruitful discussions and the referees for helpful comments. Part of the work has been done when J.-M. Yuan was visiting the Mathematics Institute of Academia Sinica, Taiwan. J.-M. Yuan thanks Professors I-Liang Chern, Jyh-Hao Lee, and ChiKun Lin for their encouragements and helpful discussions. J. Shen and J. Wu thank the National Center for Theoretical Sciences at Taipei, the Mathematics Department of National Taiwan University and the Applied Mathematics Department of Providence University, Taiwan, for support and hospitality during part of this collaboration.

\section{References}

1. Beale, J.T.: Exact solitary water waves with capillary ripples at infinity. Commun. Pure Appl. Math. 44, 211-247 (1991)

2. Boyd, J.P.: Weakly non-local solitons for capillary-gravity waves: fifth-degree Korteweg-de Vries equation. Physica D 48, 129-146 (1991)

3. Calvo, D.C., Akylas, T.R.: Do envelope solitons radiate? J. Eng. Math. 36, 41-56 (1999)

4. Calvo, D.C., Yang, T.S., Akylas, T.R.: On the stability of solitary waves with decaying oscillatory tails. Proc. R. Soc. Lond. A 456, 469-487 (2000)

5. Cui, S.-B., Deng, D.-G., Tao, S.-P.: Global existence of solutions for the Cauchy problem of the Kawahara equation with $L^{2}$ initial data. Acta Math. Sin. 22, 1457-1466 (2006)

6. Grimshaw, R., Joshi, N.: Weakly nonlocal solitary waves in a singularly perturbed Korteweg-de Vries equation. SIAM J. Appl. Math. 55, 124-135 (1995)

7. Goubet, O., Shen, J.: On the dual Petrov-Galerkin formulation of the KDV equation on a finite interval. Adv. Differ. Equ. 12, 221-239 (2007)

8. Grimshaw, R., Malomed, B., Benilov, E.: Solitary waves with damped oscillatory tails: an analysis of the fifth-order Korteweg-de Vries equation. Physica D 77, 473-485 (1994)

9. Hunter, J.K., Scheurle, J.: Existence of perturbed solitary wave solutions to a model equation for water waves. Phys. D 32, 253-268 (1988)

10. Hunter, J.K., Vanden-Broeck, J.-M.: Solitary and periodic gravity-capillary waves of finite amplitude. J. Fluid Mech. 134, 205-219 (1983)

11. Kawahara, R.: Oscillatory solitary waves in dispersive media. J. Phys. Soc. Jpn. 33, 260-264 (1972)

12. Kichenassamy, S., Olver, P.J.: Existence and nonexistence of solitary wave solutions to high-order model evolution equations. SIAM J. Math. Anal. 23, 1141-1166 (1992)

13. Lo, E., Mei, C.C.: A numerical study of water-wave modulation based on a high-order nonlinear Schrödinger equations. J. Fluid Mech. 150, 395-416 (1985)

14. Pomeau, Y., Ramani, A., Grammaticos, B.: Structural stability of the Korteweg-de Vries solitons under a singular perturbation. Physica D 31, 127-134 (1988)

15. Segur, H., Kruskal, M.D.: Non-existence of small amplitude breather solutions $\phi^{4}$ theory. Phys. Rev. Lett. 58, 747-750 (1987)

16. Shen, J.: Efficient spectral-Galerkin method I. Direct solvers for second- and fourth-order equations by using Legendre polynomials. SIAM J. Sci. Comput. 15, 1489-1505 (1994)

17. Shen, J.: Efficient Chebyshev-Legendre Galerkin methods for elliptic problems. In: Ilin, A.V., Scott, R. (eds.) Proceedings of ICOSAHOM'95, Houston. J. Math., pp. 233-240 (1996)

18. Shen, J.: A new dual-Petrov-Galerkin method for third and higher odd-order differential equations: application to the KdV equation. SIAM J. Num. Anal. 41, 1595-1619 (2003)

19. Shen, J., Li-Lian, W.: Laguerre and composite Legendre-Laguerre dual-Petrov-Galerkin methods for third-order equations. Discrete Continuous Dyn. Syst. B 6, 1381-1402 (2006)

20. Sirendaoreji: New exact travelling wave solutions for the Kawahara and modified Kawahara equations. Chaos, Solitons Fractals 19, 147-150 (2004)

21. Sun, S.M.: Existence of a generalized solitary wave with positive Bond number smaller than $\frac{1}{3}$. J. Math. Anal. Appl. 156, 471-504 (1991)

22. Sun, S.M., Shen, M.C.: Exponentially small estimate for the amplitude of capillary ripples of a generalized solitary wave. J. Math. Anal. Appl. 172, 533-566 (1993)

23. Szegö, G.: Orthogonal Polynomials. AMS Coll. Publ. (1939)

24. Vanden-Broeck, J.-M.: Elevation solitary waves with surface tension. Phys. Fluids A 3, 2659-2663 (1991) 